\title{
Impact of Phlebotomy Tourniquet Use on Blood Lactate Levels in Acutely III Patients
}

\author{
Vamsi Balakrishnan*; John Wilson*; Brent Taggart*; James Cipollat; Rebecca Jeanmonod*
}

\begin{abstract}
Objective: Lactate levels are increasingly used to guide resuscitation efforts. Some surgical literature suggests that tourniquet use during phlebotomy falsely elevates results, although studies in healthy volunteers have not demonstrated this. The purpose of this study was to determine in clinical practice whether tourniquet use during the drawing of a lactate results in significantly altered levels compared to the result of a level drawn without a tourniquet.

Methods: A prospective cohort study was carried out on emergency department patients whose clinical presentation led a physician to order a lactate level. Written informed consent was obtained from patients or their proxies. Study lactates were obtained using a tourniquet during the draw sequence of other laboratory studies. Lactate levels for clinical use were drawn per hospital protocol with no tourniquet. The time of lactate measurements and patient demographic information were recorded. Lactate levels for each patient were compared with the Wilcoxon RankSum Test.

Results: 40 patients were consented and enrolled. The median clinical lactate level was 1.9 (interquartile range 1.5-2.6), and the median study lactate level was 1.9 (interquartile range 1.4-2.7). There was no difference between paired lactate values $(p=0.95)$.

Conclusions: Tourniquet use appears to have no impact on measured lactate levels. Our findings suggest that current practices at many institutions regarding lactate collection are likely too stringent and should be changed.
\end{abstract}

\section{RÉSUMÉ}

Objectif: Le taux de lactate sert de plus en plus à guider les efforts de réanimation. D'après certains travaux de recherche en chirurgie, I'utilisation du garrot durant la ponction veineuse augmenterait erronément le taux de lactate, bien que certaines études réalisées chez des témoins en bonne santé ne soient pas arrivées à la même conclusion. L'étude décrite ici avait donc pour but de déterminer si l'utilisation du garrot en clinique durant les prélèvements de sang en vue de la mesure du taux de lactate avait pour effet de modifier considérablement le taux par rapport aux prélèvements de sang effectués sans garrot.

Méthode: Une étude de cohorte, prospective a été menée chez des patients traités au service des urgences dont le tableau clinique justifiait, selon le médecin, une mesure du taux de lactate. Les malades ou leur représentant ont donné par écrit leur consentement éclairé. Les prélèvements de sang faits pour la mesure du taux de lactate aux fins de l'étude ont été effectués avec garrot, en même temps que ceux faits pour d'autres examens de laboratoire. Les prélèvements de sang faits pour la mesure du taux de lactate aux fins cliniques ont été effectués sans garrot, selon le protocole en vigueur à I'hôpital. Ont été consignées l'heure de la mesure des taux de lactate ainsi que des données démographiques sur les patients. II y a eu comparaison des taux de lactate de chaque patient selon le test de Wilcoxon.

Résultats: Quarante patients ont participé à l'étude après avoir donné leur consentement. Le taux médian de lactate, mesuré aux fins cliniques était de 1,9 (intervalle interquartile : $1,5-2,6)$ et le taux médian de lactate, mesuré aux fins de l'étude était de 1,9 (intervalle interquartile : 1,4-2,7). Aucun écart n'a donc été enregistré entre les valeurs appariées de lactate $(p=0,95)$.

Conclusions: II semble donc que l'utilisation du garrot n'ait aucune incidence sur la mesure du taux de lactate. Les résultats de l'étude portent à croire que les pratiques en vigueur dans bon nombre d'établissements en ce qui concerne les prélèvements de sang pour la mesure du taux de lactate sont trop restrictives et qu'il faudrait les changer.

Keywords: blood lactate, tourniquet use, phlebotomy

\section{INTRODUCTION}

Lactate was first demonstrated to be present in the blood of acutely ill septic women in $1843 . .^{1,2}$ In the 1960 s, lactate was noted to be a predictor of poor outcomes in hospitalized patients. ${ }^{3}$ Additional research has shown blood lactate to be one of the most useful

From the *Department of Emergency Medicine, St. Luke's University Hospital, Bethlehem, PA, USA; tDepartment of Trauma/Critical Care, St. Luke's University Hospital, Bethlehem, PA, USA.

Correspondence to: Rebecca Jeanmonod, Department of Emergency Medicine, St. Luke's University Hospital, 801 Ostrum St., Bethlehem, PA 18015, USA. Email: rebeccajeanmonod@yahoo.com 
markers to risk stratify and guide resuscitation in critically ill patients. ${ }^{2,4}$

Debate exists in clinical practice regarding how best to collect blood for lactate measurement. Since lactate levels increase as a result of tissue hypoperfusion, it has been hypothesized that application of a tourniquet to facilitate phlebotomy may in fact elevate blood lactate levels by reducing blood flow to the affected extremity. Using a microdialysis catheter implanted directly into the quadriceps muscle, Korth and colleagues found that after induction of ischemia with a tourniquet, lactate increased linearly over time (15-75 minutes) to $206 \%$ of basal values. ${ }^{5}$ In orthopedic studies utilizing tourniquet for hemostasis, lactate levels have been found to increase; however, these studies also used tourniquet times far in excess of what is applied for a blood draw. ${ }^{6-10}$

As a result of these studies as well as due to the theoretical reasoning for tourniquet avoidance, many hospitals have protocols in place indicating that lactate levels must be drawn without a tourniquet. Some manufacturers of lactate assays as well as the Surviving Sepsis Campaign Bundle also recommend drawing lactates without tourniquets. ${ }^{11,12}$ Phlebotomy without the use of a tourniquet is technically more difficult as a result of blood vessels' smaller diameter and reduced turgor. ${ }^{13}$ This increase in difficulty may lead to failure to obtain a lactate level or a delay in the time necessary to obtain this important test.

In healthy volunteers, tourniquet use has not been found to increase blood lactate significantly with tourniquet times up to 15 minutes, or with blood pressure cuffs elevated to mean arterial pressure for a duration of five minutes. ${ }^{14,15}$ However, the effect of tourniquet use on lactate levels in clinical practice has not been studied. The purpose of this study was to determine in clinical practice whether tourniquet use during the drawing of a lactate results in significantly altered levels compared to the result of a level drawn without a tourniquet.

\section{MATERIALS AND METHODS}

\section{Study design}

A prospective cohort study was undertaken of patients presenting to the emergency department (ED) for whom physicians ordered a lactate level as part of clinical care. The reasons for physicians drawing clinical lactates included evaluation of suspected sepsis or ischemic bowel. Blood samples from these patients were utilized for both the clinical lactate and the study lactate. Participation in this study resulted in no additional cost, change in standard care, harm, or benefit to the patients enrolled, as the attending physicians did not have access to study results.

\section{Study setting and population}

This study was carried out at St. Luke's University Hospital in Bethlehem, Pennsylvania, a level 1 community trauma center with an ED census of 60,000. A convenience sample of adult patients ( $\geq 18$ years) were screened for eligibility over a one year period in 2013-2014, based on availability of research associates to complete the informed consent process with the patients. Patients were deemed eligible if the attending physician ordered a lactate during their clinical care. Trauma patients were excluded. Participants were enrolled after written informed consent was obtained from the patient or health care proxy. The study was approved by the institutional review board at the study hospital.

\section{Study protocol and measurements}

Lactate levels for clinical use were drawn as per standard protocol at the study hospital with no tourniquet used during collection. Study lactates were obtained using a tourniquet during the draw sequence of other laboratory studies ordered at the discretion of the treating provider. If consent was obtained prior to establishment of intravenous access, the study lactate was typically drawn last in the draw sequence of other blood studies during intravenous catheter placement. If the consent was obtained after blood studies had already been drawn, the study lactate was typically drawn during a second venipuncture for a second blood culture. There were no circumstances in which the patient underwent a blood draw solely for the purpose of obtaining a study lactate.

Clinical lactate levels were reported in the electronic laboratory result system as per hospital standard and were available to physicians while patient care was ongoing. Study lactate levels were stripped of patient identifiers and coded for blinding purposes. Results of these assays were mailed to the research investigators as private correspondence and were not reported in the medical record or used for patient care. 
The hospital lactate assay was performed utilizing a Flex Reagent Cartridge in a Dimension Clinical Chemistry System analyzer with an analytical sensitivity of $<0.3 \mathrm{mmol} / \mathrm{L}$ (Siemens Healthcare Diagnostics, NY).

The times of clinical lactate and study lactate draw were recorded, as were patient age and gender. These were entered into a standardized Microsoft Excel 2007 spreadsheet (Microsoft Corporation, Redmond, WA) by trained research associates.

\section{Data analysis}

Because the clinical and study lactates represent repeated measures in single patients, the two values were compared with Wilcoxon Signed-Rank test. This statistic was used because it was expected that the two lactates would be drawn by two methods at close to the same time. We did not use a T-test, because we had an a priori expectation that the lactate levels would display skew, with tail toward higher values clustering around the normal range. Linear correlation was used to assess overall relationship between clinical and study lactate. Demographic information was reported with standard descriptive statistics. Data were analyzed using VassarStats: Website for Statistical Computation (vassarstats.net, author Richard Lowry, PhD, Professor of Psychology Emeritus, Vassar College, Poughkeepsie, NY, (C) 1998-2013).

\section{RESULTS}

Forty patients were enrolled over the one year study period. The mean age of study participants was 72.7 years (standard of deviation 16.4 years), and $62.5 \%$ were male. Enrolled patients were typically ill and undergoing evaluation for infectious processes (sepsis) or intra-abdominal surgical disease (mesenteric ischemia or other surgical disease).

Clinical lactate and study lactate were drawn a median of six minutes apart (IQR 3-20 minutes, range $0-151$ minutes). The lactate reference range for the laboratory performing the study was $0.5-2.2 \mathrm{mmol} / \mathrm{L}$. For the patients enrolled in this study, the median clinical lactate level was $1.9 \mathrm{mmol} / \mathrm{L}$ (IQR 1.5-2.6, range $0.7-5.6)$. The median study lactate level was $1.9 \mathrm{mmol} / \mathrm{L}$ (IQR 1.4-2.7, range $0.8-5.7$ ). $37.5 \%$ of clinical lactate levels fell above the reference standard $(\mathrm{n}=15)$.

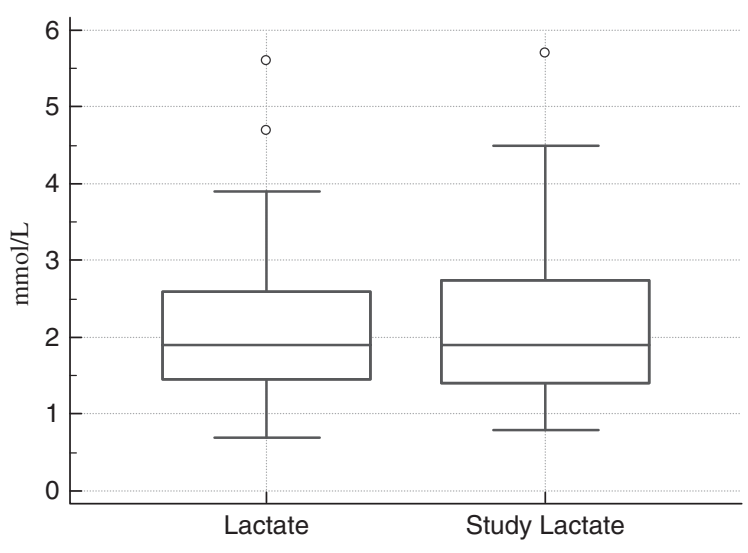

Figure 1. Comparison of clinical lactate levels to study lactate levels drawn with a tourniquet.

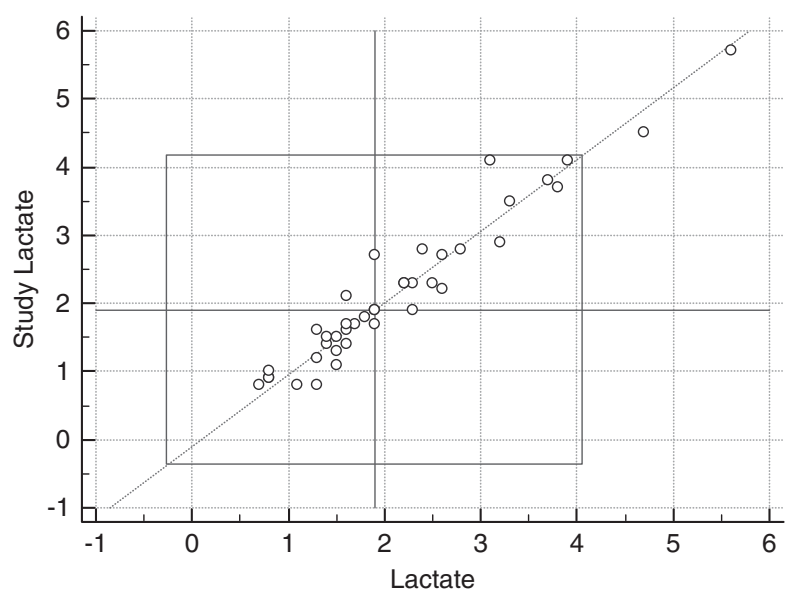

Figure 2. Relationship of standard draw (clinical) lactate levels to study lactate levels (drawn with a phlebotomy tourniquet) in $\mathrm{mmol} / \mathrm{L}$.

Using a two-tailed Wilcoxon Rank-Sum test, there was no statistical difference found between clinical and study lactate levels ( $p=0.95$, Figure 1$)$. Considering only the 15 patients with clinical lactate levels above the reference standard, there were no differences in clinical and study lactate levels $(p=0.96)$. On simple linear correlation, the correlation coefficient between the clinical and study lactate was 0.97 (CI 0.94-0.98) with an r-square of 0.93 (Figure 2).

\section{DISCUSSION}

Accurate and timely measurement of lactate is important. Our findings suggest that current practices at many institutions regarding lactate collection are likely too stringent and should be changed. These practices appear to have been put in place based on extrapolation from 
orthopedic and anesthesia research; however, studies of that nature involved higher tourniquet pressures and longer periods of time than that used in routine phlebotomy.

In routine phlebotomy, the standard of care is to apply a tourniquet that is tight enough to cause target vein dilation (i.e., tight enough to occlude venous flow), but not so tight as to cause arterial obstruction. If increasing pressures are responsible for increasing lactate in tourniquet patients, as some have suggested, ${ }^{10}$ it may be that the disposable tourniquets used in phlebotomy simply do not generate enough pressure to elevate lactate. If this is the case, situations in which phlebotomy is performed using a blood pressure cuff for target vein dilation may result in different blood lactate results depending on the level to which the cuff is inflated. Prior surgical studies generally involved increasing the tourniquet pressure to above the mean arterial pressure (MAP) in order to ensure a bloodless field during extremity surgery. ${ }^{6-10}$ These results may indicate that blood pressure cuff increase to above the MAP could also falsely increase lactate values in clinical practice; however, this has not been adequately studied.

In addition to lower pressure, phlebotomy tourniquets are usually applied for less time than those involved in surgical studies. In standard phlebotomy practice, tourniquet up-times are usually less than a minute, and the World Health Organization indicates a tourniquet up-time of two minutes or less is best practice. $^{16,17}$ We did not record tourniquet up-time, but suspect that, since the study lactate was always the last blood drawn (so as not to interfere with necessary studies for patient management), study values represent lactates taken at the upper limit of tourniquet time. In a prior study of healthy volunteers, even 15 minutes of tourniquet up-time with a phlebotomy tourniquet did not alter lactate levels. ${ }^{14}$ Our study was not designed to find a specific tourniquet time beyond which blood lactate might be falsely elevated, but within the constraints of our methodology we found no evidence that lactate was affected by routine tourniquet application.

Our patient population was medically ill, and typically being evaluated for sepsis. We did not enroll trauma patients, although lactate levels are often used to guide resuscitative efforts during trauma. Elevated lactate levels in both trauma and sepsis are secondary to perfusion mismatch. As a result, we suspect that phlebotomy tourniquet use would not falsely elevated lactate levels in such patients, but our study was not adequate to determine impact of phlebotomy tourniquet use in all patients in whom a lactate might be obtained.

\section{LIMITATIONS}

Although our patients were all patients for whom a physician deemed a lactate level would be clinically useful, there are limitations introduced in any sample of this nature. Patients needed to be conscious and competent in order to consent to participate, or alternatively have a valid power of attorney accompanying them. Very ill patients are not always awake and alert, and clinicians do not usually approach the critically ill for research purposes due to the importance of timely resuscitation and treatment. As a result, the patients in whom this study might be most applicable (i.e., extremely ill septic patients) may not have been adequately represented in our study population. The population from which the lactate levels were measured was primarily made up of those who were not in extremis, but who were patients for whom it was judged by the attending physician involved that a lactate would be useful.

In addition, we did not attempt to standardize or record tourniquet up-time in study patients. It is possible that for some patients, for whom intravenous access was difficult to obtain, there were more prolonged tourniquet times in order to increase the success of phlebotomy. Since we did not standardize or record these times, we cannot evaluate the impact of time of tourniquet use on lactate levels.

We did not capture the clinical scenarios involved in the decision by treating physicians to order a lactate level. There may be differences in tourniquet effect based upon underlying patient comorbidities or the cause of an elevated lactate (i.e., "Type A," secondary to perfusion mismatch, versus "Type B," secondary to inability to clear the lactate).

Finally, our study was performed at a single institution with a small number of participants arising from convenience sampling, and thus may not be generalizable to other settings and populations. As a result of our methods, a selection bias for less sick patients existed, as our study could not result in delayed care for critically ill patients.

\section{CONCLUSIONS}

Tourniquet use appears to have no impact on measured lactate levels. Our findings suggest that current practices 
at many institutions regarding lactate collection are likely too stringent and should be changed.

Competing Interests: None declared.

\section{REFERENCES}

1. Kompanje EJ, Jansen TC, Van der hoven B, et al. The first demonstration of lactic acid in human blood in shock by Johann Joseph Scherer (1814-1869) in January 1843. Intensive Care Med 2007;33(11):1967-71.

2. Fuller BM, Dellinger RP. Lactate as a hemodynamic marker in the critically ill. Curr Opin Crit Care 2012;18(3):267-72.

3. Huckabee WE. Abnormal resting blood lactate. I. The significance of hyperlactatemia in hospitalized patients. Am 7 Med 1961;30(6):833-9.

4. Dellinger RP, Levy MM, Rhodes A, et al. Surviving sepsis campaign: international guidelines for management of severe sepsis and septic shock: 2012. Crit Care Med 2013;41(2):580-637.

5. Korth U, Merkel G, Fernandez FF, et al. Tourniquetinduced changes of energy metabolism in human skeletal muscle monitored by microdialysis. Anesthesiology 2000; 93(6):1407-12.

6. Kang HJ, Han CD, Jahng JS, et al. Blood gas and electrolyte changes after tourniquet application in total knee replacement surgery. Yonsei Med $\mathcal{F}$ 1992;32(2):153-8.

7. Karalezli N, Ogun CO, Ogun TC, et al. Wrist tourniquet the most patient-friendly way of bloodless hand surgery. 7 Trauma 2007;62(3):750-4.

8. Matejec R, Schulz A, Harbach HW, et al. Effects of tourniquet-induced ischemia on the release of proopiomelanocortin derivatives determined in peripheral blood plasma. 7 Appl Physiol 2004;97(3):1040-5.
9. Kokki H, Väätäinen U, Penttilä I. Metabolic effects of a low-pressure tourniquet system compared with a highpressure tourniquet system in arthroscopic anterior crucial ligament reconstruction. Acta Anaesthesiol Scand 1998; 42(4):418-24.

10. Chalidis BE, Kalivas E, Parziali $M$, et al. Cuff width increases the serum biochemical markers of tourniquetinduced skeletal muscle ischemia in rabbits. Orthopedics 2012;35(8):e1245-50.

11. Procedure manual for Abbott i-STAT system. Available at: www.abbottpointofcare.com/Customer-Info-Center (accessed February 24, 2015).

12. Society of Critical Care Medicine. Surviving Sepsis Campaign 3-Hour Bundle; 2015. Available at: http://www. survivingsepsis.org/SiteCollectionDocuments/Bundle-3-HourStep1-Measure-Lactate.pdf (accessed February 24, 2015).

13. Sasaki S, Murakami N, Matsumura Y, et al. Relationship between tourniquet pressure and a cross-section area of superficial vein of forearm. Acta Med Okayama 2012; 66(1):67-71.

14. Jones AE, Leonard MM, Hernandez-Nino J, et al. Determination of the effect of in vitro time, temperature, and tourniquet use on whole blood venous point-of-care lactate concentrations. Acad Emerg Med 2007;14(7):587-91.

15. Bany-Mohammed FM, Macknin ML, van Lente F, et al. The effect of prolonged tourniquet application on serum bicarbonate. Cleve Clin 7 Med 1995;62(1):68-70.

16. Serdar MA, Kenar L, Hasimi A, et al. Tourniquet application time during phlebotomy and the influence on clinical chemistry testing; is it negligible? Turk 7 Biochem 2008; 33(3):85-8.

17. World Health Organization (WHO). WHO guidelines on drawing blood: best practices in phlebotomy; 2010. Available at: whqlibdoc.who.int/publications/2010/9789241599221_eng. pdf (accessed February 25, 2015). 